\begin{tabular}{|c|l|}
\hline Title & Ratio Mercerian theorems with applications to Hankel and Fourier transforms \\
\hline Author(s) & Bingham, N. H; Inoue, A. \\
\hline Citation & Hokkaido University Preprint Series in Mathematics, 395, 1-30 \\
\hline Issue Date & 1997-10-1 \\
\hline DOI & 10.14943/83541 \\
\hline Doc URL & http://hdl.handle.net/2115/69145 \\
\hline Type & bulletin (article) \\
\hline File Information & pre395.pdf \\
\hline
\end{tabular}

Instructions for use 


\section{RATIO MERCERIAN THEOREMS WITH APPLICATIONS TO HANKEL AND FOURIER TRANSFORMS}

N. H. Bingham and A. Inoue

Series \#395. October 1997 


\section{HOKKADO UNIVERSITY PREPRINT SERIES IN MATHEMATICS}

\#370 N.H. Bingham and A. Inoue, An Abel-Tauber theorem for Hankel transforms, 8 pages. 1997.

\#371 T. Nakazi and H. Sawada, The commutator ideal in Toeplitz algebras for uniform algebras and the analytic structure, 9 pages. 1997.

\#372 M.-H. Giga and Y. Giga, Stability for evolving graphs by nonlocal weighted curvature, 70 pages. 1997.

\#373 T. Nakazi, Brown-Halmos type theorems of weighted Toeplitz operators, 14 pages. 1997.

\#374 J. Inoue and S.-E. Takahashi, On characterizations of the image of Gelfand transform of commutative Banach algebras, 30 pages. 1997.

\#375 L. Solomon and H. Terao, The double coxeter arrangement, 21 pages. 1997.

\#376 G. Ishikawa and T. Morimoto, Solution surfaces of Monge-Ampère equations, 15 pages. 1997.

\#377 G. Ishikawa, A relative transversality theorem and its applications, 16 pages. 1997.

\#378 J. Inoue and T. Nakazi, On the zeroes of solutions of an extremal problem in $H^{1}, 14$ pages. 1997.

\#379 Y. Giga and K. Ito, On pinching of curves moved by surface diffusion, 12 pages. 1997.

\#380 F. Hiroshima, Weak coupling limit removing an ultraviolet cut-off for a Hamiltonian of particles interacting with a scalar field, 39 pages. 1997.

\#381 Y. Giga, S. Matsui and Y. Shimizu, On estimates in Hardy spaces for the Stokes flow in a half space, 13 pages. 1997.

\#382 A. Arai, A new estimate for the ground state energy of Schrödinger operators, 12 pages. 1997.

\#383 M. Nakamura and T. Ozawa, The Cauchy problem for nonlinear wave equations in the Sobolev space of critical order, 24 pages. 1997.

\#384 K. Ito, Asymptotic stability of planar rarefaction wave for scalar viscous conservation law, 8 pages. 1997.

\#385 A. Arai, Representation-theoretic aspects of two-dimensional quantum systems in singular vector potentials: canonical commutation relations, quantum algebras, and reduction to lattice quantum systems, 32 pages. 1997.

\#386 P. Aviles and Y. Giga, On lower semicontinuity of a defect energy obtained by a singular limit of the Ginzburg-Landau type energy for gradient fields, 21 pages. 1997.

\#387 T. Nakazi and T. Yamamoto, Norms of some singular integral operators and their inverse operators, 28 pages. 1997.

\#388 M.-H. Giga and Y. Giga, Remarks on convergence of evolving graphs by nonlocal curvature, 18 pages. 1997.

\#389 T. Tsukada, Reticular Lagrangian singularities, 41 pages. 1997.

\#390 M. Nakamura and T. Ozawa, The Cauchy problem for nonlinear wave equations in the homogeneous Sobolev space, 12 pages. 1997.

\#391 Y. Giga, M. Ohnuma and M.-H. Sato, On strong maximum principle and large time behaviour of generalized mean curvature flow with the Neumann boundary condition, 24 pages. 1997.

\#392 T. Tsujishita and H. Watanabe, Monoidal closedness of the category of simulations, 24 pages. 1997.

\#393 T. Arase, A remark on the quantale structure of multisets, 10 pages. 1997.

\#394 N. H. Bingham and A. Inoue, Extension of the Drasin-Shea-Jordan theorem, 16 pages. 1997. 


\title{
RATIO MERCERIAN THEOREMS WITH APPLICATIONS TO HANKEL AND FOURIER TRANSFORMS
}

\author{
N. H. BINGHAM and A. INOUE
}

\section{$\S 1$. Introduction.}

Tauberian theorems have an extensive literature and a long history; broadly speaking, one can distinguish between the works of Hardy and Littlewood and their predecessors on special kernels, and of Wiener (from 1932 on) and his successors on general kernels. Ratio Tauberian theorems have formed a small sub-area of this topic; for background and references, see e.g. [2], §2.10.1. Mercerian theorems, though much less studied than Tauberian theorems, are closely linked to them, and have an analogous history. Specialkernel results go back to Hardy in 1912 and before, general-kernel results to Paley and Wiener [18] in 1933 (see Chapter IV of their classic book [19]). Subsequent textbook accounts are in [21], Ch. V, [2], Ch. 5. The particular Mercerian theorems studied here are of Drasin-Shea-Jordan type (see below for details and references). This study completes and complements our recent work [3] on Drasin-Shea-Jordan theorems for Fourier and Hankel transforms. In improving on the methods of [3], we were led to the ratio Mercerian theorems below (Theorems 1-3 of §2) for general kernels; these yield definitive versions of our earlier results for Fourier and Hankel transforms (Theorems 4-6 of $\S 2$ and Corollary).

For $\rho \in \mathbf{R}$, we write $R_{\rho}$ for the class of functions $f$ regularly varying (at infinity) with index $\rho$ : $f$ is measurable, positive for large enough $x$, and

$$
f(\lambda x) / f(x) \rightarrow \lambda^{\rho} \quad(x \rightarrow \infty) \quad \forall \lambda>0 ;
$$

see [2] for background. We are concerned here with comparisons between the asymptotic behaviour of $f$ and that of integral transforms of $f$. We will write these in Mellinconvolution form as

$$
(k * f)(x):=\int_{0}^{\infty} f(t) k(x / t) d t / t \quad(x>0),
$$

for suitable kernels $k$. The simplest results of this type are Abelian, and state that under suitable conditions

$$
f(x) \sim x^{\rho} \ell(x) \quad(x \rightarrow \infty)
$$


with $\ell$ slowly varying (i.e. $\ell \in R_{0}$ ) implies

$$
(k * f)(x) \sim c x^{\rho} \ell(x) \quad(x \rightarrow \infty)
$$

where, if the Mellin transform of the kernel $k$ is

$$
\begin{gathered}
\check{k}(s):=\int_{0}^{\infty} t^{-s} k(t) d t / t=\int_{0}^{\infty} u^{s} k(1 / u) d u / u, \\
c=\check{k}(\rho) .
\end{gathered}
$$

Tauberian results supply a partial converse, under suitable side-conditions (Tauberian conditions); see e.g. Ch. 4 of [2] for a detailed treatment.

In the circumstances above, one has

$$
(k * f)(x) / f(x) \rightarrow c \quad(x \rightarrow \infty)
$$

The question arises of whether one can obtain (1) - and so (2) - from (3), with $\rho$ such that $c=\check{k}(\rho)$. Such results are Mercerian in character. The prototypes are due to Drasin and Shea ([7], Th. 6.2; [2], Th. 5.2.1), with $k$ non-negative, and Jordan [12], where $k$ can change sign. The proofs, which are rather long, involve reduction - by real analysis and Pólya's lemma - to an integral equation, uniqueness of solution of which is guaranteed by Fourier analysis (Titchmarsh [26], Th. 146); such uniqueness questions are related to the Wiener Tauberian theory.

The Abelian and Tauberian results here say that regular variation gives comparability of function and transform, and the Mercerian result that, conversely, comparability of function and transform gives regular variation of both. Such results are of interest and importance in at least two areas: the theories of completely regular growth in complex analysis, and of domains of attraction in probability theory. In complex analysis, one links regular variation of the growth of an entire function of finite order with that of its zerocounting function, by the Levin-Pfluger theory (for details and references, see Levin [15], or [2], Ch. 7); the most basic case is that of functions with only negative zeros, studied by Valiron and Titchmarsh (see e.g. [2], §7.2). Here the relevant transform is a Stieltjes transform. The first Mercerian theorem here was proved (not under that name) by Edrei and Fuchs [8] in 1966, followed by work of Drasin [5] in 1968 and Shea [24] in 1969; in particular, Shea's paper (p. 3) contains the astute observation that such results are in fact Mercerian in character. Further results of this type were proved by Drasin and Shea in 1970 and 1976 [6, 7] and Jordan in 1974 [12]. In probability theory, domains of attraction describe the probability distributions which give rise to stable limit laws - in other words, 
which give generalizations of the central limit theorem: one draws $n$ independent copies from the distribution, adds them, then centres and scales suitably, and asks for a limit distribution. It has long been known that domains of attraction involve regular variation; see e.g. Feller [9], [2] §8.3. In the case of positive random variables, the natural transform to use (because it takes addition of independent random variables, or convolution of distributions, into products of transforms) is Laplace-Stieltjes; comparability of function (actually, the tail of the probability distribution function) and transform has long been known ([9], XIII.6,7), and that conversely this implies membership of a domain of attraction follows from the Drasin-Shea theorem ([2], Th. 5:2.4). For random variables that take both signs, however, the relevant transform is Fourier-Stieltjes (the characteristic function). Here the Abelian and Tauberian statements are due to Pitman in 1968 [20]; the Mercerian statement follows from our earlier paper [3].

The Drasin-Shea-Jordan theory as expounded in [2] uses absolute integrals and requires absolute convergence of the Mellin transform $\check{k}(s)$ (in an appropriate strip in the complex $s$-plane). However, some of the most interesting and important classical integral transforms - in particular, the Fourier sine and cosine transforms mentioned above and Hankel transforms (see also Titchmarsh [26] and Watson [27]) - have Mellin transforms which are only conditionally convergent. A Drasin-Shea-Jordan theorem for Fourier and Hankel transforms was obtained recently by the authors in [3]. Two main extra complications arise:

(i) One is compelled to make use of special properties of the kernels, and so one's results lose the generality which is one of the characteristic advantages of the Drasin-Shea-Jordan theory (and of the Wiener Tauberian theory related to it);

(ii) One obtains a more complicated integral equation, whose solution requires a farreaching extension of the Wiener Tauberian theory due to Nyman [17] and Korenblum [13], [14]; see Gurarii [11] for an accessible treatment in English rather than Russian.

This paper removes a third difficulty which arises in [2], the restriction to Hankel transforms of small order. There, we use the kernel $k$ given by

$$
k(x):=x^{-3 / 2} J_{\nu}(1 / x)
$$

with $J_{\nu}$ the Bessel function of order $\nu \geq-1 / 2$. We write $F_{\nu}$, or simply $F$, for the Hankel transform of $f$, defined in Mellin-convolution form as

$$
F(x):=\int_{0}^{\infty} f(t)(t / x)^{3 / 2} J_{\nu}(t / x) d t / t \quad(x>0) .
$$

Note that the Fourier cosine and sine transforms are the special cases $\nu=-1 / 2,1 / 2$, as

$$
J_{-1 / 2}(z)=\sqrt{\frac{2}{\pi}} \frac{\cos z}{\sqrt{z}}, \quad J_{-1 / 2}(z)=\sqrt{\frac{2}{\pi}} \frac{\sin z}{\sqrt{z}},
$$


also that the Mellin transform is given by Weber's integral:

$$
a_{\nu}(s):=\check{k}(s)=\int_{0}^{\infty} t^{-s} k(t) d t / t=2^{s+\frac{1}{2}} \frac{\Gamma\left(\frac{3}{4}+\frac{\nu}{2}+\frac{s}{2}\right)}{\Gamma\left(\frac{1}{4}+\frac{\nu}{2}-\frac{s}{2}\right)} \quad\left(-\nu-\frac{3}{2}<\Re s<\nu+\frac{1}{2}\right)
$$

(Watson [27], 13.24 (1), Titchmarsh [26], (7.4.1): the integral is defined in the first instance in the strip $-\nu-\frac{3}{2}<\Re s<0$, but extends to the larger strip by analytic continuation). The results of [3] are restricted to 'small' $\nu: \nu \leq \nu_{0}$, where $\nu_{0} \in\left(\frac{1}{2}, 1\right)$ is the unique root of the transcendental equation

$$
\log 2+\frac{\Gamma^{\prime}\left(\frac{\nu_{0}}{2}+\frac{1}{2}\right)}{\Gamma\left(\frac{\nu_{0}}{2}+\frac{1}{2}\right)}=0
$$

$\left(\nu_{0}=0.8660252365 \ldots:\right.$ thus the cosine and sine cases are included in $\left.\nu \leq \nu_{0}\right)$.

Here we remove the restriction $\nu \leq \nu_{0}$, so covering all $\nu \geq-\frac{1}{2}$; we simplify the proofs by a localization technique (discussed in $\S 2$ below); we obtain a new type of 'ratio Mercerian theorem' (Theorems 1-3 below); and we apply our results to Hankel transforms (Theorems 4, 5) and characteristic functions in probability theory (Theorem 6 and its Corollary).

Recall that the upper order $\rho(f)$ of an ultimately positive function $f$ is defined by

$$
\rho(f):=\limsup \operatorname{sum}_{x \rightarrow \infty} \log f(x) / \log x
$$

(thus if $f \in R_{\rho}, \rho(f)=\rho$ ). The localization technique and the ratio Mercerian results allow us avoid the condition

$$
-\nu-\frac{3}{2}<\rho(f)<0
$$

needed in [3], as a hypothesis.

\section{§. Results.}

First we state three 'ratio Mercerian' theorems, which hold for general transforms rather than for special cases such as Hankel transforms. We need to recall $([2], \S 2.1 .2)$ the Matuszewska indices of a positive function $f$. The upper Matuszewska index $\alpha(f)$ is the infimum of those $\alpha$ for which there exists a constant $C=C(\alpha)$ such that for each $\Lambda>1$,

$$
f(\lambda x) / f(x) \leq C(1+o(1)) \lambda^{\alpha} \quad(x \rightarrow \infty) \text { uniformly in } \lambda \in[1, \Lambda]
$$

the lower Matuszewska index $\beta(f)$ is the supremum of those $\beta$ for which, for some constant $D=D(\beta)>0$ and all $\Lambda>1$,

$$
f(\lambda x) / f(x) \geq D(1+o(1)) \lambda^{\beta} \quad(x \rightarrow \infty) \text { uniformly in } \lambda \in[1, \Lambda] .
$$


One says $f$ has bounded increase, $f \in B I$, if $\alpha(f)<\infty$, bounded decrease, $f \in B D$, if $\beta(f)>-\infty$.

$$
=
$$

THEOREM 1. Let $-\infty<a<b \leq \infty$. For $i=1,2$, let $k_{i}:(0, \infty) \rightarrow(0, \infty)$ be nonnegative measurable functions such that the Mellin transforms $\check{k}_{i}(z)$ converge absolutely for $a<\Re z<b$. We assume

$$
\int_{0}^{\infty} k_{1}(t) d t / t^{1+a}<\infty, \quad \int_{0}^{\infty} k_{2}(t) d t / t^{1+a}=\infty .
$$

Let $f$ be a non-negative measurable function such that $f$ is locally bounded on $[0, \infty)$, vanishes in a neighbourhood of zero, has finite upper order $\rho:=\rho(f) \in[a, b)$, and $f \in$ $B D \cup B I$. If

$$
\frac{\left(k_{2} * f\right)(x)}{\left(k_{1} * f\right)(x)} \rightarrow c \in[0, \infty) \quad(x \rightarrow \infty)
$$

then $\rho>a$.

THEOREM 2. For non-negative measurable $k_{1}:(0, \infty) \rightarrow[0, \infty)$ and measurable $k_{2}$ : $(0, \infty) \rightarrow \mathbf{R}$, let the Mellin transforms $\check{k}_{1}(z)$ and $\breve{k}_{2}(z)$ both converge absolutely in the strip $a<\Re z<b$. Let $f$ be a non-negative measurable function, locally bounded on $[0, \infty)$, vanishing near zero, of finite order $\rho \in(a, b)$, and with $f \in B D \cup B I$. If

$$
\frac{\left(k_{2} * f\right)(x)}{\left(k_{1} * f\right)(x)} \rightarrow c \neq 0 \quad(x \rightarrow \infty)
$$

then $c=\check{k}_{2}(\rho) / \check{k}_{1}(\rho)$.

THEOREM 3. In Theorem 2, we write

$$
k_{0}(x):=\check{k}_{2}(\rho) k_{1}(x)-\check{k}_{1}(\rho) k_{2}(x) \quad(0<x<\infty)
$$

and assume that

$$
\check{k}_{0}(z) \text { has a unique zero } z=\rho \text { on the vertical line } \Re z=\rho \text {, }
$$

and that this zero is at most double. We also assume

$$
\frac{\log \left|\check{k}_{0}(\rho+i y)\right|}{\exp \left(\frac{\pi|y|}{2 \epsilon_{0}}\right)} \rightarrow 0 \quad(y \rightarrow \pm \infty)
$$


for some $\epsilon_{0}>0$. Then $E_{2} * k_{1} * f \in R_{\rho}$ with $E_{2}(x):=I_{(0,1]}(x) \cdot x^{\rho+\epsilon}$ for sufficiently small $\epsilon>0$.

Of course, our interest is in regular variation of $f$ itself, rather than of a smoothed version such as $E_{2} * k_{1} * f$ as here. However, we will be able to apply the theorems above to Hankel transforms to obtain definitive versions of the results of [3].

THEOREM 4. Let $\nu \geq-1 / 2, t^{\nu+\frac{1}{2}} f(t) \in L_{\text {loc }}^{1}[0, \infty), f$ be ultimately decreasing to zero at infinity with upper order $\rho$ and Hankel transform $F$. If

$$
\int_{0}^{\infty} t^{\nu+\frac{1}{2}} f(t) d t \neq 0 \quad \text { or } \quad \rho>-\nu-\frac{3}{2}
$$

and

$$
F(x) / f(x) \rightarrow c \neq 0 \quad(x \rightarrow \infty)
$$

then

(i) $-1<\rho<0$ if $\nu=-1 / 2$, and $-\nu-\frac{3}{2}<\rho \leq 0$ if $\nu>-1 / 2$;

(ii) $c=a_{\nu}(\rho)$.

THEOREM 5. In Theorem 4, we further assume $\rho \neq-\frac{1}{2}$. Then $f \in R_{\rho}$.

For non-negative $f$, Theorems 4 and 5 together achieve the 'ideal form' like that for, say, the Laplace transform ([2], Th. 5.2.4). But there one has a positive kernel with an absolutely convergent transform, and here our kernels change sign and have only conditionally convergent transforms, which makes matters much harder.

We comment briefly on the conditions in Theorems 4 and 5 .

a. The counter-example of [3] $\S 7$ shows that the condition $\rho \neq-\frac{1}{2}$ in Theorem 5 is essential. Now by results of [3] (Propositions 1 of $\S 4$ and 5 of $\S 7$ ), for Hankel transforms $\rho \neq-\frac{1}{2}$ is equivalent to

$$
\check{k}(\rho+i \tau) \neq \check{k}(\rho) \quad(\tau \in \mathbf{R}, \quad \tau \neq 0)
$$

(we imposed this condition on $k_{0}$ as $(2.3)$ in Theorem 3 - note that $\check{k}_{0}(\rho)=0$ ). Condition (*) has been used by Ganelius [10] and Jordan ([12], (2.4)) in general-kernel Mercerian theorems; they also showed by counter-example $([10],[12], \S 4)$ that this condition is essential.

In [3], for Hankel kernels, we needed not only $(*)$ itself but also its counter-part 
of 'strip' form because we needed to use Korenblum's theorem. In [3], we could check the condition (*) of strip form by virtue of the explicit form of $\check{k}$ (see (W)). Now in this paper, in which we apply Theorem 3 to prove Theorem 5 , checking $(*)$ is replaced by checking (2.3). Since we also use Korenblum's theorem in the proof of Theorem 3, we need the strip form of (2.3). However, in the case of Theorem 3 where $\check{k}_{0}$ is defined by an absolutely convergent integral, the strip form of (2.3) follows from (2.3) itself automatically by the Riemann-Lebesgue Lemma and Vitali's theorem (see the beginning of the proof of Theorem 3 below as well as [2], §5.1.3).

Now $\rho$ is the upper order of $f$, and we may lack a priori information on $f$ allowing us to check $\rho \neq-\frac{1}{2}$ in Theorem 5 conveniently. In practice, what we do have a priori information on is the comparison constant $c$; this is linked to $\rho$ via $c=\check{k}(\rho)$, as in $\S 1$. Now by Weber's integral $(\mathrm{W}), \check{k}\left(-\frac{1}{2}\right)=1$. For $\nu \leq \nu_{0}, \rho=-\frac{1}{2}$ is the only real root of $\check{k}(s)=1$ (by the monotonicity result of [3], Proposition 3), so in the context of [3] we may replace $\rho \neq-\frac{1}{2}$ in Theorem 5 by $c \neq 1$, obtaining an equivalent reformulation of Theorem 5. However, for $\nu>\nu_{0}$, there are three roots (see the Proposition of $\S 5$ ). We may still reformulate Theorem 5 by replacing $\rho \neq-\frac{1}{2}$ by $c \neq 1$, but as this is now a stronger condition we obtain a weaker theorem.

b. The condition that $f$ be ultimately decreasing ensures that the integral giving the Hankel transform converges (conditionally). Some such condition is needed. For less stringent conditions of this type (such as quasi-monotonicity), see e.g. [2], §4.3; we use monotonicity for simplicity. This implies $\rho \leq 0$.

c. In our earlier work [3], the extra condition $-\nu-\frac{3}{2}<\rho(f)<0$ is assumed; it ties the order of $f$ to the convergence-strip of the Mellin transform of the kernel. It is avoided in Theorems 4 and 5 under the condition $A \neq 0$, where $A:=\int_{0}^{\infty} t^{\nu+\frac{1}{2}} f(t) d t$. We note that the latter condition $\rho>-\nu-\frac{3}{2}$ in (2.5) implies $A=\infty$, hence the former condition $A \neq 0$ (see [3], §3). The condition $A \neq 0$ is essential; in $\S 8$, we give a counter-example showing this.

d. The condition $\nu \leq \nu_{0}$ in [3] concerns one of the main points of the present paper, so we discuss it in detail. For a generic kernel $k$, let $\{z \in \mathbf{C}: \Re z \in(a, b)\}$ be the (absolute or conditional) convergence strip of $\breve{k}$ (thus $(a, b)=\left(-\nu-\frac{3}{2}, \nu+\frac{1}{2}\right)$ in our case $\left.k(x)=x^{-3 / 2} J_{\nu}(1 / x)\right)$, and $\rho$ be the upper order of $f$. The major difference between this paper and its predecessors, by Drasin and Shea [7], Jordan [12] and the authors [3], is that we dispense with the following condition:

$$
\check{k} \text { is monotone on either }(a, \rho] \text { or }[\rho, b) \text {, }
$$

which is needed for the method of proof used there. In fact, for Hankel transforms with 
$\nu>\nu_{0}$, there appears a subinterval $\left(b_{1}, b_{2}\right) \subset(a, b)$ such that for $\rho \in\left(b_{1}, b_{2}\right)$ the above condition (A) is not satisfied (see $\S 5$ ). For those $\nu \in\left[-\frac{1}{2}, \nu_{0}\right]$ considered in [3], $\check{k}$ is strictly decreasing on $(a, b)$, whence (A) holds for any $\rho \in(a, b)$. In [12], Theorems 1 and $1 a$, Jordan explicitly assumes (A), while in [7], where $k \geq 0,(\mathrm{~A})$ is automatically satisfied since $\breve{k}^{\prime \prime}>0$ and $\breve{k}$ is convex. Here we adopt a new method of proof, a type of localization, which enables us to dispense with both condition (A) and use of a key tool in [7], [12] and [3], the Pólya Peak Theorem of Drasin and Shea, and thereby greatly to simply the proofs. This method also enables us to avoid the assumption $-\nu-\frac{3}{2}<\rho<0$. It may be found in the proofs of Theorems 1 and 3.

The above Theorems 1, 2 and 3 have other applications. For example, we have

THEOREM 6. Let $f(t) \in L_{\text {loc }}^{1}[0, \infty), f$ be ultimately decreasing to zero at infinity. Let $F_{\mathrm{c}}, F_{\mathrm{s}}$ be the Fourier cosine and sine transforms of $f$, respectively: for $x>0$,

$$
F_{\mathrm{c}}(x):=\int_{0}^{\infty-} f(t) \cos t x d t, \quad F_{\mathrm{s}}(x):=\int_{0}^{\infty-} f(t) \sin t x d t
$$

If

$$
\int_{0}^{\infty} f(t) d t \neq 0
$$

and

$$
F_{\mathrm{c}}(x) / F_{\mathrm{B}}(x) \rightarrow c \neq 0 \quad(x \rightarrow 0+),
$$

then $c>0$ and $f \in R_{\rho}$ with $\rho:=-(2 / \pi) \arctan c \in(-1,0)$.

We apply Theorem 6 to the tail behaviour of a random variable. Let $X$ be a non-negative random variable and let $T$ be its tail probability:

$$
T(x):=P(X>x) \quad(x \geq 0)
$$

Then $T(x)$ is bounded on $[0, \infty)$ and decreasing to zero as $x \rightarrow \infty$. Let $U$ and $V$ be the real and imaginary parts of the characteristic function of $X$ :

$$
U(t):=E[\cos t X], \quad V(t):=E[\sin t X]
$$

Then

$$
\{1-U(t)\} / t=\int_{0}^{\infty-} T(x) \sin t x d x
$$




$$
V(t) / t=\int_{0}^{\infty} T(x) \cos t x d x
$$

(see, e.g., [2], p. 336). By Theorem 6, we immediately obtain the following

COROLLARY. Let $T, U$ and $V$ be as above. If

$$
\frac{V(t)}{1-U(t)} \rightarrow c \neq 0 \quad(t \rightarrow 0+)
$$

then $c>0$ and $T \in R_{\rho}$ with $\rho:=-(2 / \pi) \arctan c \in(-1,0)$.

\section{§3. Korenblum's theorem.}

It is convenient in this section to work with additive convolutions and Fourier transforms, and to use $k$ for a generic kernel (not the Bessel kernel of $\S 1$ ); when we apply our results later, we will revert to using multiplicative convolutions and Mellin transforms. We write the Fourier transform as

$$
\hat{k}(z):=\frac{1}{\sqrt{2 \pi}} \int_{-\infty}^{\infty} k(x) e^{-i x z} d x .
$$

Recall first the key result of the Wiener Tauberian theory for $L_{1}(\mathbf{R})$, giving the equivalence of three statements:

(i) $\hat{k}(t)$ has no zeros $t \in \mathbf{R}_{\text {; }}$

(ii) linear combinations of translates of $k$ are dense in $L_{1}$;

(iii) the only solutions $g \in L_{\infty}(\mathbf{R})$ of the integral equation

$$
(k * g)(x):=\int_{-\infty}^{\infty} k(x-y) g(y) d y \equiv 0
$$

are the trivial ones $g=0$ a.e.

For background to this Wiener approximation theorem, see e.g. Reiter [23] I, §4.1, Bingham [1]. In particular, the resulting theory extends to finitely many real zeros $z_{j}, j=1, \cdots, r$ (or finitely many common zeros for classes of kernels). The non-trivial solutions $g$ are now linear combinations of the $\exp \left\{i x z_{j}\right\}(n=1, \cdots, r)$; in fact, this is equal to saying that the finite subset $\left\{z_{j}\right\}$ of $\mathbf{R}$ is a Wiener set with respect to the pair $\left(L_{1}(\mathbf{R}), L_{\infty}(\mathbf{R})\right.$ ) (see, e.g., $[23], \mathrm{I}, \S 4.7)$.

The extension of this theory from $\left(L_{1}, L_{\infty}\right)$ to other pairs of function spaces in 
duality is due to Nyman and Korenblum; see e.g. Gurarii [11], Borichev [4], [2] §4.8.5. For $\alpha>0$, one replaces $L_{1}(\mathbf{R})$ by the Banach algebra $L(\alpha)$ of functions $k$ with norm

$$
\|k\|:=\int_{-\infty}^{\infty}|k(x)| e^{\alpha|x|} d x
$$

and convolution as multiplication. The dual space $L(\alpha)^{*}$ of $L(\alpha)$ is the space of measurable functions $g$ with norm

$$
\|g\|:=\operatorname{ess}-\sup \left\{|g(x)| e^{-\alpha|x|}: x \in \mathbf{R}\right\}<\infty
$$

the duality is given by

$$
\langle k, g\rangle:=\int_{-\infty}^{\infty} k(x) g(x) d x \quad\left(k \in L(\alpha), g \in L(\alpha)^{*}\right) .
$$

For $k \in L(\alpha)$, set

$$
\gamma^{ \pm}(k):=\lim \sup _{x \rightarrow \pm \infty}\left(\exp \left\{\frac{-\pi|x|}{2 \alpha}\right\} \log |\hat{k}(x)|\right)
$$

$\left(\hat{k}(x) \rightarrow 0\right.$ as $x \rightarrow \pm \infty$ by the Riemann-Lebesgue lemma, so $\left.\gamma^{ \pm}(k) \leq 0\right)$. The Fourier transform $\hat{k}(z)$ is now defined in the strip $|\Im z| \leq \alpha$ in the complex $z$-plane. The analogue for $L(\alpha)$ of the Wiener condition that $\hat{k}$ be non-vanishing on $\mathbf{R}$ (except at finitely many zeros) is the non -vanishing of $\hat{k}$ in the strip (except at finitely many zeros $z_{j}$ of multiplicity $n_{j}$ ), and in addition Korenblum's condition

$$
\gamma^{+}(k)=\gamma^{-}(k)=0
$$

which says that $\hat{k}$ should not decay to zero at infinity in the strip too fast. The Wiener results on non-trivial solutions $g \in L_{\infty}(\mathbf{R})$ to the integral equation (iii) now extend to $g \in L(\alpha)^{*}$. We shall need the case $r=1, n_{1}=1$ or 2 of one simple or double zero of $\hat{k}$ below.

\section{$\S 4$. Proofs of Theorems 1,2 and 3.}

Proof of Theorem 1. For $\gamma \in(\rho, b)$, there exists $d(\gamma) \in(0, \infty)$ such that

$$
f(x) \leq d(\gamma) x^{\gamma} \quad(0<x<\infty)
$$


By the following crude but useful bound

$$
|K * f(x)| \leq d(\gamma) \cdot|K| \Upsilon(\gamma) x^{\gamma} \quad(0<x<\infty)
$$

both $k_{1} * f(x)$ and $k_{2} * f(x)$ converge absolutely for $x>0$.

By Lemma 7 of [3] and the remark just after the proof of Theorem 5.2.3 of [2], the assumption $f \in B \cdot D \cup B I$ yields

$$
\int_{0}^{\infty} f(t) d t / t^{1+\gamma}=\infty \quad(\gamma<\rho)
$$

whence by the Fubini-Tonelli theorem

$$
\int_{0}^{\infty} k_{1} * f(t) d t / t^{1+\gamma}=\check{k}_{1}(\gamma) \check{f}(\gamma)=\infty \quad(\gamma<\rho) .
$$

For small $\epsilon>0$, we write $p_{1}:=\rho-\epsilon, p_{2}:=\rho+\epsilon$ and consider

$$
E_{1}(x):=I_{(1, \infty)}(x) x^{p_{1}}, \quad E_{2}(x):=I_{(0,1)}(x) x^{p_{2}}
$$

We write

$$
g(x):=\left(E_{1} * E_{2} * f\right)(x) \quad(0<x<\infty) .
$$

The integral converges by repeated use of the crude bound above.

By (2.1) and (4.2),

$$
\frac{\left(E_{1} * k_{2} * f\right)(x)}{\left(E_{1} * k_{1} * f\right)(x)}=\frac{\int_{0}^{x} k_{2} * f(t) d t / t^{1+p_{1}}}{\int_{0}^{x} k_{1} * f(t) d t / t^{1+p_{1}}} \rightarrow c \quad(x \rightarrow \infty)
$$

(see Step 3 of the proof of Theorem 5.2.1 of [2]), and so

$$
\frac{k_{2} * g(x)}{k_{1} * g(x)}=\frac{\int_{x}^{\infty}\left(E_{1} * k_{2} * f\right)(t) d t / t^{1+p_{2}}}{\int_{x}^{\infty}\left(E_{1} * k_{1} * f\right)(t) d t / t^{1+p_{2}}} \rightarrow c \quad(x \rightarrow \infty)
$$

Since $x^{-p_{1}} E_{1}(x)$ is increasing and

$$
x^{-p_{1}} g(x)=\int_{0}^{\infty}(x / t)^{-p_{1}} E_{1}(x / t)\left(E_{2} * f\right)(t) d t / t^{1+p_{1}}
$$

$x^{-p_{1}} g(x)$ is increasing. Similarly, $x^{-p_{2}} g(x)$ is decreasing. Therefore

$$
\left(k_{1} * g\right)(x)=x^{p_{2}} \int_{0}^{1} \frac{k_{1}(t)}{t^{1+p_{2}}}\left(\frac{x}{t}\right)^{-p_{2}} g(x / t) d t+x^{p_{1}} \int_{1}^{\infty} \frac{k_{1}(t)}{t^{1+p_{1}}}\left(\frac{x}{t}\right)^{-p_{1}} g(x / t) d t
$$




$$
\leq g(x)\left(\int_{0}^{1} \frac{k_{1}(t)}{t^{1+p_{2}}} d t+\int_{1}^{\infty} \frac{k_{1}(t)}{t^{1+p_{1}}} d t\right)
$$

Similarly

$$
\left(k_{2} * g\right)(x) \geq g(x) \int_{1}^{\infty} \frac{k_{2}(t)}{t^{1+p_{2}}} d t
$$

So

$$
c=\lim _{x \rightarrow \infty} \frac{\left(k_{2} * g\right)(x)}{\left(k_{1} * g\right)(x)} \geq \frac{\int_{1}^{\infty} k_{2}(t) d t / t^{1+\rho+\epsilon}}{\int_{0}^{1} k_{1}(t) d t / t^{1+\rho+\epsilon}+\int_{1}^{\infty} k_{1}(t) d t / t^{1+\rho-\epsilon}} .
$$

However, if $\rho=a$, then the right-hand side tends to infinity as $\epsilon \downarrow 0$ since

$$
\int_{0}^{\infty} \frac{k_{1}(t)}{t^{1+a}} d t<\infty, \quad \int_{1}^{\infty} \frac{k_{2}(t)}{t^{1+a}} d t=\infty
$$

This is a contradiction, whence $\rho>a$.

Proof of Theorem 2. For $\gamma \in(\rho, b)$, there exists $d(\gamma) \in(0, \infty)$ such that

$$
f(x) \leq d(\gamma) x^{\gamma} \quad(0<x<\infty)
$$

We write

$$
F_{i}(x):=\left(k_{i} * f\right)(x) \quad(0<x<\infty, \quad i=1,2) .
$$

Then by the bound

$$
\left|F_{i}(x)\right| \leq d(\gamma) \cdot\left|k_{i}\right|^{\sim}(\gamma) \cdot x^{\gamma} \quad(0<x<\infty)
$$

we have for any $X>0$ and $i=1,2$,

$$
\begin{array}{ll}
\int_{0}^{X}\left|F_{i}(t)\right| d t / t^{1+\gamma}<\infty & (\gamma<b) \\
\int_{X}^{\infty}\left|F_{i}(t)\right| d t / t^{1+\gamma}<\infty & (\rho<\gamma) .
\end{array}
$$

By Fubini's theorem,

$$
\left(k_{2} * F_{1}\right)(x)=\left(k_{2} *\left(k_{1} * f\right)\right)(x)=\left(k_{1} *\left(k_{2} * f\right)\right)(x)=\left(k_{1} * F_{2}\right)(x)
$$

or

$$
\left(k_{2} * F_{1}\right)(x)-\left(k_{1} * F_{2}\right)(x)=0 \quad(0<x<\infty)
$$


Again by Fubini's theorem,

$$
\left(k_{2} * F_{1}\right)^{\circ}(z)=\check{k}_{2}(z) \check{F}_{1}(z), \quad\left(k_{1} * \bar{F}_{2}\right)^{\circ}(z)=\check{k}_{1}(z) \check{F}_{2}(z) \quad(\rho<\Re z<b),
$$

whence by (4.6)

$$
\check{k}_{1}(z) \check{F}_{2}(z)-\check{k}_{2}(z) \check{F}_{1}(z)=0 \quad(\rho<\Re z<b) .
$$

Clearly $k_{1}$ is not identically equal to zero, so that $\check{k}_{1}(\rho)>0$. Therefore one has, for $z$ sufficiently close to $\rho$ with $\Re z>\rho$,

$$
\int_{0}^{\infty}\left\{F_{2}(t)-\frac{\check{k}_{2}(z)}{\check{k}_{1}(z)} F_{1}(t)\right\} d t / t^{1+z}=0
$$

By (4.1), Fubini's theorem yields

$$
\int_{0}^{\infty} F_{1}(t) d t / t^{1+\gamma}=\check{F}_{1}(\gamma)=\left(k_{1} * f\right)^{-}(\gamma)=\check{k}_{1}(\gamma) \check{f}(\gamma)=\infty \quad(\gamma<\rho)
$$

which with (4.4) gives, for $X>0$,

$$
\int_{X}^{\infty} F_{1}(t) d t / t^{1+\gamma}=\infty \quad(\gamma<\rho)
$$

Therefore, in the same way as Step 1 of the proof of Theorem 5.2.1 of [2], we can use Pólya's lemma (cf. [2], Lemma 5.2.2) to show $c \leq \check{k}_{2}(\rho) / \check{k}_{1}(\rho)$. Similarly, by a method similar to Step 2 of the proof of Theorem 5.3.1 of [2], $c \geq \check{k}_{2}(\rho) / \check{k}_{1}(\rho)$, whence $c=\check{k}_{2}(\rho) / \check{k}_{1}(\rho)$.

Proof of Theorem 3. By the Riemann-Lebesgue Lemma and Vitali's theorem in complex analysis (see e.g. Titchmarsh [25], §5.2), the condition (2.3) enables us to take $\epsilon \in\left(0, \epsilon_{0}\right)$ such that $[\rho-\epsilon, \rho+\epsilon] \subset(a, b)$ and that $\check{k}_{0}(z)$ has a unique root $z=\rho$ in the strip $\rho-\epsilon \leq \Re z \leq \rho+\epsilon$ (see [2], §5.1.3, Jordan [10], p.191). This root is at most double by assumption. Since $\epsilon_{0}<\epsilon,(2.4)$ also holds with $\epsilon_{0}$ replaced by $\epsilon$.

Let $p_{1}:=\rho-\epsilon, p_{2}:=\rho+\epsilon$ and $E_{1}, E_{2}, F_{1}, F_{2}$ be as above. We write

$$
h_{i}(x):=\left(E_{2} * E_{1} * F_{i}\right)(x) \quad(0<x<\infty, \quad i=1,2) .
$$

Then the crude bound above allows use of Fubini's theorem to transform (4.6) into

$$
\left(k_{2} * h_{1}\right)(x)-\left(k_{1} * h_{2}\right)(x)=0 \quad(0<x<\infty)
$$


By (4.8) and (2.2),

$$
\frac{\left(E_{1} * F_{2}\right)(x)}{\left(E_{1} * F_{1}\right)(x)}=\frac{\int_{0}^{x} F_{2}(t) d t / t^{1+p_{1}}}{\int_{0}^{x} F_{1}(t) d t / t^{1+p_{1}}} \rightarrow c \quad(x \rightarrow \infty)
$$

and so

$$
\frac{h_{2}(x)}{h_{1}(x)}=\frac{\int_{x}^{\infty}\left(E_{1} * F_{2}\right)(t) d t / t^{1+p_{2}}}{\int_{x}^{\infty}\left(E_{1} * F_{1}\right)(t) d t / t^{1+p_{2}}} \rightarrow c \quad(x \rightarrow \infty) .
$$

Since $x^{-p_{1}} h_{i}(x)$ is increasing and $x^{-p_{2}} h_{i}(x)$ is decreasing, one has

$$
h_{i}(u x) / h_{i}(x) \leq \max \left(u^{p_{1}}, u^{p_{2}}\right) \quad(0<x<\infty, \quad i=1,2) .
$$

Now choose any sequence $x_{n} \uparrow \infty$. Consider

$$
j_{n}(u):=h_{1}\left(x_{n} u\right) / h_{1}\left(x_{n}\right) \quad(u>0)
$$

By the Helly selection principle, we can find a sequence of integers $n^{\prime} \uparrow \infty$ such that $j_{n^{\prime}}(u)$ converges pointwise on $(0, \infty)$, to $j$, say. Then $u^{-p_{1}} j(u)$ is increasing, $j(1)=1$, and by (4.12)

$$
j(u) \leq \max \left(u^{p_{1}}, u^{p_{2}}\right) \quad(0<u<\infty) .
$$

By (4.11), $h_{2}\left(x_{n^{\prime}} u\right) / h_{2}\left(x_{n^{\prime}}\right) \rightarrow j(u)$ as $n^{\prime} \rightarrow \infty$.

From (4.10),

$$
\frac{h_{2}\left(x_{n^{\prime}}\right)}{h_{1}\left(x_{n^{\prime}}\right)} \int_{0}^{\infty} \frac{h_{2}\left(x x_{n^{\prime}} / t\right)}{h_{2}\left(x_{n^{\prime}}\right)} k_{1}(t) d t / t-\int_{0}^{\infty} \frac{h_{1}\left(x x_{n^{\prime}} / t\right)}{h_{1}\left(x_{n^{\prime}}\right)} k_{2}(t) d t / t=0 \quad(0<x<\infty) .
$$

We now have suitably dominated convergence of both integrands. So

$$
\frac{\check{k}_{2}(\rho)}{\check{k}_{1}(\rho)} \int_{0}^{\infty} j(x / t) k_{1}(t) d t / t-\int_{0}^{\infty} j(x / t) k_{2}(t) d t / t=0 \quad(0<x<\infty),
$$

or simply

$$
\left(k_{0} * j\right)=0 \quad(0<x<\infty)
$$

To apply Korenblum's theory, let

$$
\phi(x):=j\left(e^{x}\right) e^{-\rho x}, \quad K(x):=k_{0}\left(e^{x}\right) e^{-\rho x} \quad(x \in \mathbf{R}) .
$$

Then

$$
\phi(x) \leq \max \left(e^{-\epsilon x}, e^{\epsilon x}\right)=e^{\epsilon|x|}
$$


and

$$
\int_{-\infty}^{\infty} e^{\epsilon|x|}|K(x)| d x<\infty
$$

Our integral equation is

$$
\int_{-\infty}^{\infty} K(x-y) \phi(y) d y=0 \quad(\forall x \in \mathbf{R})
$$

The Fourier transform $\hat{K}$ of $K$ is equal to $(2 \pi)^{-1 / 2} \check{k}_{0}(\rho+i z)$ for $|\Im z| \leq \epsilon$. So by the strip form of assumption (2.3), $\hat{K}(z)$ has a unique root in the strip $|\Im z| \leq \epsilon$, which is at most double. Since we have (2.4), Korenblum's theorem now yields

$$
\phi(x)=a_{1}+a_{2} x \quad \text { a.e. } \quad x \in \mathrm{R}
$$

for some $a_{1}, a_{2} \in \mathrm{C}$. That is,

$$
j(x) x^{-\rho}=a_{1}+a_{2} \log x \quad \text { a.e. } \quad x \in \mathbf{R} .
$$

Since $j$ is real, so are $a_{1}, a_{2}$. Since $x^{-p_{1}} j(x)$ is increasing, $j(1-)=a_{1}=j(1+)$, whence $a_{1}=1$. Since $j(\cdot) \geq 0, a_{2}=0$. So $j(x)=x^{\rho}$ a.e.; this and $x^{-p_{1}} j(x)$ increasing give

$$
j(x)=x^{p} \quad(\forall x>0)
$$

This says that the partial limit $u^{\rho}$ of $h_{1}\left(x_{n} u\right) / h_{1}\left(x_{n}\right)$ does not depend on the particular sequence $\left(x_{n}\right)$ chosen. Thus

$$
h_{1}(x u) / h_{1}(x) \rightarrow u^{\rho} \quad(x \rightarrow \infty) \quad \forall u>0,
$$

and so $h_{1} \in R_{\rho}$.

Since $x^{-p_{2}} E_{2} * F_{1}(x)$ is decreasing, the function $\log \left(x^{-\left(1+p_{1}\right)} E_{2} * F_{1}(x)\right)$ is slowly increasing. So we can use a monotone density argument on

$$
h_{1}(x)=E_{1} * E_{2} * F_{1}(x)=x^{p_{1}} \int_{x}^{\infty} t^{-\left(1+p_{1}\right)} E_{2} * F_{1}(t) d t
$$

to pass from $h_{1} \in R_{\rho}$ to $E_{2} * F_{1}=E_{2} * k_{1} * f \in R_{\rho}$, as required.

§5. The Mellin transform $a_{\nu}$.

Write

$$
\psi(x):=\Gamma^{\prime}(x) / \Gamma(x)
$$


for the logarithmic derivative of the gamma function (the digamma function). Recall the series expansions

$$
\psi(z)=-\gamma-\frac{1}{z}+z \sum_{n=1}^{\infty} \frac{1}{n(z+n)}, \quad \psi^{\prime}(z)=\sum_{n=0}^{\infty} 1 /(z+n)^{2}
$$

([28], $\S 12.16$, or [16], p. 14). Thus $\psi$ is increasing, and $\psi^{\prime}$ decreasing, on $(0, \infty)$. As above, define $\nu_{0} \in\left(\frac{1}{2}, 1\right)$ by

$$
\log 2+\psi\left(\frac{\nu_{0}}{2}+\frac{1}{2}\right)=0
$$

PROPOSITION. For $\nu>\nu_{0}$, there exist $b_{1}$ and $b_{2}$ with

$$
-\nu-\frac{3}{2}<b_{1}<-\frac{1}{2}<b_{2}<\nu+\frac{1}{2}
$$

such that $a_{\nu}$ decreases on $\left(-\nu-\frac{3}{2}, b_{1}\right)$, increases on $\left(b_{1}, b_{2}\right)$ and decreases on $\left(b_{2}, \nu+\frac{1}{2}\right)$, with $a_{\nu}^{\prime \prime}\left(b_{1}\right)>0$ and $a_{\nu}^{\prime \prime}\left(b_{2}\right)<0$.

Proof. For $x \in\left(-\nu-\frac{3}{2}, \nu+\frac{1}{2}\right)$, write

$$
f_{\nu}(x):=\log a_{\nu}(x)=\left(x+\frac{1}{2}\right) \log 2+\log \Gamma\left(\frac{x}{2}+\frac{\nu}{2}+\frac{3}{4}\right)-\log \Gamma\left(-\frac{x}{2}+\frac{\nu}{2}+\frac{1}{4}\right)
$$

Then

$$
\begin{gathered}
f_{\nu}^{\prime}(x)=\log 2+\frac{1}{2} \psi\left(\frac{x}{2}+\frac{\nu}{2}+\frac{3}{4}\right)+\frac{1}{2} \psi\left(-\frac{x}{2}+\frac{\nu}{2}+\frac{1}{4}\right), \\
f_{\nu}^{\prime \prime}(x)=\frac{1}{4} \psi^{\prime}\left(\frac{x}{2}+\frac{\nu}{2}+\frac{3}{4}\right)-\frac{1}{4} \psi^{\prime}\left(-\frac{x}{2}+\frac{\nu}{2}+\frac{1}{4}\right) .
\end{gathered}
$$

Since $\psi(x) \rightarrow-\infty$ as $x \downarrow 0$ (from the series expansion),

$$
f_{\nu}^{\prime}(x) \rightarrow-\infty \quad \text { as } \quad x \downarrow-\nu-\frac{3}{2} \quad \text { or } \quad x \uparrow \nu+\frac{1}{2} \text {. }
$$

Also

$$
f_{\nu}^{\prime}\left(-\frac{1}{2}\right)=\log 2+\psi\left(\frac{\nu}{2}+\frac{1}{2}\right)>0
$$

as $\nu>\nu_{0}$, and since $\psi^{\prime}$ decreases, $f_{\nu}^{\prime \prime}$ is positive on $\left(-\infty,-\frac{1}{2}\right)$, zero at $-\frac{1}{2}$ and negative on $\left(-\frac{1}{2}, \infty\right)$. Consequently, there exist $b_{1}, b_{2}$ in the ranges specified in the Proposition with

$$
f_{\nu}^{\prime}\left(b_{1}\right)=f_{\nu}^{\prime}\left(b_{2}\right)=0
$$

By considering the second derivative, we can easily check that the signs of the second derivatives at these points are as stated. 
COROLLARY. For $\nu \geq-\frac{1}{2}, \lambda \in\left(-\nu-\frac{3}{2}, \nu+\frac{1}{2}\right) \backslash\left\{-\frac{1}{2}\right\}$, the root $z=\lambda$ of $a_{\nu}(z)-a_{\nu}(\lambda)$ is at most double.

\section{§6. Proofs of Theorems 4 and 5.}

As in [3], we introduce the positive kernels defined by

$$
C(x):=x^{\nu+\frac{1}{2}} e^{-x}, \quad D(x):=d_{\nu} \frac{x^{\nu+\frac{3}{2}}}{\left(1+x^{2}\right)^{\nu+\frac{3}{2}}},
$$

where

$$
d_{\nu}:=\pi^{-\frac{1}{2}} 2^{\nu+1} \Gamma\left(\nu+\frac{3}{2}\right) .
$$

The convergence strips of their Mellin transforms $\check{C}, \check{D}$ are $\Re z<\nu+\frac{1}{2},-\nu-\frac{3}{2}<\Re z<\nu+\frac{3}{2}$, respectively. By a formula of Gegenbauer (see [27], $\$ 13.2(5)$ ),

$$
D(x)=(k * C)(x) \quad(0<x<\infty)
$$

where $k(x):=x^{-3 / 2} J_{\nu}(1 / x)$ is the kernel of Hankel transform in $\S 1$.

Choose $X>0$ so large that $f$ is finite and decreasing on $[X, \infty)$. We write $\tilde{f}(x):=I_{[X, \infty)} f(x)$. In the proof below, we first prove

$$
\frac{D * \tilde{f}(x)}{C * \tilde{f}(x)} \rightarrow c \quad(x \rightarrow \infty),
$$

and then apply Theorems 1,2 and 3 .

Step 1. We show $D * f=C * F$ :

$$
d_{\nu} x^{\nu+\frac{3}{2}} \int_{0}^{\infty} \frac{t^{\nu+\frac{1}{2}}}{\left(x^{2}+t^{2}\right)^{\nu+\frac{3}{2}}} f(t) d t=\int_{0}^{\infty}(x / t)^{\nu+\frac{1}{2}} e^{-x / t} F(t) d t / t \quad(0<x<\infty),
$$

both integrals converging absolutely. In Lemma 2 of [3], we proved (6.2) under the assumption $\rho<0$. By the same proof, which makes essential use of the Parseval formula for Hankel transforms, we find that (6.2) also holds even without $\rho<0$ if we replace $\int_{0}^{\infty}$ on the right by $\int_{0}^{\infty-}$. However, $F(x)$ is of constant sign for $x$ large enough by (2.6), whence $\int_{0}^{\infty-}$ again reduces to $\int_{0}^{\infty}$. Thus (6.2) follows. In particular, together with (2.6), this gives

$$
\int_{a}^{\infty} \frac{|F(x)|}{x^{\nu+\frac{3}{2}}} d x<\infty, \quad \int_{a}^{\infty} \frac{|f(x)|}{x^{\nu+\frac{3}{2}}} d x<\infty \quad(\forall a>0) .
$$


Step 2. We prove (6.1). First we prove

$$
\int_{0}^{\infty} t^{\nu+\frac{1}{2}} f(t) d t=\infty
$$

For simplicity, we write $A$ for the integral on the left. Since the assumption $\rho>-\nu-\frac{3}{2}$ implies (6.4) as in [3], $\S 3$, it is enough to prove (6.4) under the condition $A \neq 0$. Suppose $A$ is non-zero but finite. Since there exists a positive constant $M$ such that

$$
\left|x^{-\nu} J_{\nu}(x)\right|<M \quad(0<x<\infty)
$$

the dominated convergence theorem yields

$$
\begin{aligned}
& x^{\nu+\frac{3}{2}} f(x)=\frac{f(x)}{F(x)} \int_{0}^{\infty} t^{\nu+\frac{1}{2}} f(t)(t / x)^{-\nu} J_{\nu}(t / x) d t \\
& \left.\rightarrow \frac{A}{c 2^{\nu} \Gamma(\nu+1)} \neq 0 \quad \text { (hence }>0\right) \quad(x \rightarrow \infty) .
\end{aligned}
$$

But this implies $A=\infty$, which contradicts our assumption. Thus we also obtain (6.4) under the condition $A \neq 0$. By (6.4),

$$
\frac{D * f(x)}{D * \tilde{f}(x)}=1+\frac{\int_{0}^{X} t^{\nu+\frac{1}{2}} f(t) d t /\left(1+(t / x)^{2}\right)^{\nu+\frac{3}{2}}}{\int_{X}^{\infty} t^{\nu+\frac{1}{2}} f(t) d t /\left(1+(t / x)^{2}\right)^{\nu+\frac{3}{2}}} \rightarrow 1 \quad(x \rightarrow \infty) .
$$

Now, by (6.3), we may consider $C * \tilde{f}$. So as in [3], §3, we have

$$
\frac{D * f(x)}{C * \tilde{f}(x)}=\frac{C * F(x)}{C * \tilde{f}(x)} \rightarrow c \quad(x \rightarrow \infty) .
$$

Combining, we obtain (6.1). We also find $c>0$, by (6.1) and the assumption $c \neq 0$.

Step 3. We prove Theorem 4. Since $f$ is eventually decreasing, $\rho \leq 0$, while $\rho \geq-\nu-\frac{3}{2}$ by (6.4). So $-\nu-\frac{3}{2} \leq \rho \leq 0$.

Suppose $\nu=-1 / 2$ but $\rho=0$. For $\epsilon \in(0,1)$ and $p_{1}:=-\epsilon, p_{2}:=\epsilon$, let $E_{1}, E_{2}$ be as in $\S 4$. Since

$$
C * \tilde{f}(x)=\int_{0}^{\infty} e^{-x / t} \tilde{f}(t) d t / t \leq \int_{X}^{\infty} f(t) d t / t \quad(0<x<\infty)
$$

(6.3) shows that $C * \tilde{f}$ is bounded. Therefore we may consider $E_{2} * E_{1} * C * \tilde{f}$, and similarly $E_{2} * E_{1} * D * \tilde{f}$. As in (4.3), we have

$$
\frac{D * g(x)}{C * g(x)} \rightarrow c \quad(x \rightarrow \infty)
$$


where

$$
g(x):=E_{1} * E_{2} * \tilde{f}(x) \quad(0<x<\infty) .
$$

Since $x^{\epsilon} g(x)$ is increasing and $x^{-\epsilon} g(x)$ is decreasing,

$$
\begin{gathered}
D * g(x)=x^{\epsilon} \int_{0}^{1} \frac{D(t)}{t^{1+\epsilon}}(x / t)^{-\epsilon} g(x / t) d t+x^{-\epsilon} \int_{1}^{\infty} \frac{D(t)}{t^{1-\epsilon}}(x / t)^{\epsilon} g(x / t) d t \\
\leq g(x)\left(\int_{0}^{1} \frac{D(t)}{t^{1+\epsilon}} d t+\int_{1}^{\infty} \frac{D(t)}{t^{1-\epsilon}} d t\right) .
\end{gathered}
$$

Similarly,

$$
C * g(x) \geq g(x) \int_{0}^{1} \frac{C(t)}{t^{1-\epsilon}} d t
$$

Therefore

$$
\frac{1}{c}=\lim _{x \rightarrow \infty} \frac{(C * g)(x)}{(D * g)(x)} \geq \frac{\int_{0}^{1} C(t) d t / t^{1-\epsilon}}{\int_{0}^{1} D(t) d t / t^{1+\epsilon}+\int_{1}^{\infty} D(t) d t / t^{1-\epsilon}} .
$$

However, the right-hand side tends to infinity as $\epsilon \downarrow 0$ since

$$
\int_{0}^{1} C(t) d t / t=\int_{0}^{1} e^{-t} d t / t=\infty, \quad \int_{0}^{\infty} D(t) d t / t=\sqrt{\frac{2}{\pi}} \int_{0}^{\infty} \frac{1}{\left(1+t^{2}\right)} d t<\infty
$$

giving a contradiction. Thus $\rho \neq 0$ if $\nu=-1 / 2$.

Since $\rho \in[-\nu-3 / 2, \nu+1 / 2)$ and

$$
\int_{0}^{\infty} t^{\nu+\frac{1}{2}} C(t) d t<\infty, \quad \int_{0}^{\infty} t^{\nu+\frac{1}{2}} D(t) d t=\infty
$$

we can apply Theorem 1 to obtain $\rho>-\nu-\frac{3}{2}$, whence Theorem 4 (i). Since $-\nu-\frac{3}{2}<$ $\rho<\nu+\frac{1}{2}$, we can apply Theorem 2 to obtain $c=\check{D}(\rho) / \check{C}(\rho)$. But

$$
\begin{gathered}
\check{C}(z)=\int_{0}^{\infty} t^{\nu-z-\frac{1}{2}} e^{-t} d t=\Gamma\left(-z+\nu+\frac{1}{2}\right) \quad\left(\Re z<\nu+\frac{1}{2}\right), \\
\check{D}(z)=\int_{0}^{\infty} \frac{t^{\nu+\frac{1}{2}-z}}{\left(1+t^{2}\right)^{\nu+\frac{3}{2}}} d t=\Gamma\left(-z+\nu+\frac{1}{2}\right) a_{\nu}(z) \quad\left(-\nu-\frac{3}{2}<\Re z<\nu+\frac{3}{2}\right),
\end{gathered}
$$

whence $c=a_{\nu}(\rho)$. Thus Theorem 4 (ii) follows.

Step 4. We prove Theorem 5. By Proposition 1 of [3], $a_{\nu}(z)$ takes the value $a_{\nu}$ only at $z=\rho$ on the line $\Re z=\rho$. If we write

$$
k_{0}(x):=\check{D}(\rho) C(x)-\check{C}(\rho) D(x) \quad(x>0),
$$


then the Mellin transform $\check{k}_{0}$ of $k_{0}$ has the strip of absolute convergence $-\nu-\frac{3}{2}<\Re z<$ $\nu+\frac{1}{2}$, and is given there by

$$
\check{k}_{0}(z)=\Gamma\left(-\rho+\nu+\frac{1}{2}\right) \Gamma\left(-z+\nu+\frac{1}{2}\right)\left\{a_{\nu}(\rho)-a_{\nu}(z)\right\} .
$$

So $\check{k}_{0}(z)$ has a unique root $z=\rho$ on the line $\Re z=\rho$ ), which by the Corollary to the Proposition in $\S 5$ is at most double. By Propositions 2 and 4 of [3] - or by direct calculation from the complex form of Stirling's formula (see e.g. [22], p. 38 or [28], §12.33) - the Korenblum condition (2.4) of Theorem 3 is satisfied for some $\epsilon_{0}>0$. Therefore $E_{2} * C * \tilde{f} \in$ $R_{\rho}$ by Theorem 3 , for small enough $\epsilon>0$.

We conclude as in [3], $\S 6$, Step 6 . Since

$$
C * \tilde{f}(x)=x^{\nu+\frac{1}{2}} \int_{0}^{\infty} e^{-x u} u^{\nu-\frac{1}{2}} \tilde{f}(1 / u) d u
$$

the function $x^{-\left(\nu+\frac{1}{2}\right)} C * \tilde{f}(x)$ is decreasing, whence $\log \left(x^{-\left(1+p_{2}\right)} C * \tilde{f}(x)\right)$ is slowly increasing. So we can apply a monotone density argument to

$$
E_{2} * C * \tilde{f}(x)=x^{p_{2}} \int_{x}^{\infty} t^{-\left(1+p_{2}\right)} C * \tilde{f}(t) d t
$$

in order to pass from $E_{2} * C * \tilde{f} \in R_{\rho}$ to $C * \tilde{f} \in R_{\rho}$. Now the integral in (6.5) is essentially a Laplace transform. The Hardy-Littlewood-Karamata theorem (or Karamata's Tauberian theorem for Laplace transforms - [2], §1.7) and a further monotone density argument give $\tilde{f} \in R_{\rho}$. This says that $f \in R_{\rho}$, as required.

§7. Proof of Theorem 6.

We write

$$
F_{1}(x):=x^{-1} F_{8}(1 / x), \quad F_{2}(x):=x^{-1} F_{\mathrm{c}}(1 / x) \quad(0<x<\infty) .
$$

Then, in Mellin convolution notation,

$$
F_{1}=K_{1} * f, \quad F_{2}=K_{2} * f,
$$

where

$$
K_{1}(x):=\frac{\sin (1 / x)}{x}, \quad K_{2}(x):=\frac{\cos (1 / x)}{x} .
$$

Our key assumption (2.8) is

$$
\frac{K_{2} * f(x)}{K_{1} * f(x)} \rightarrow c \neq 0 \quad(x \rightarrow \infty) .
$$


Now we have

$$
\int_{0}^{\infty} e^{-x t} \sin t d t=\frac{1}{x^{2}+1}, \quad \int_{0}^{\infty} e^{-x t} \cos t d t=\frac{x}{x^{2}+1} \quad(0<x<\infty)
$$

or, in Mellin-convolution notation,

$$
D_{1}(x)=\left(C * K_{1}\right)(x), \quad D_{2}(x)=\left(C * K_{2}\right)(x)
$$

where

$$
C(x):=e^{-x}, \quad D_{1}(x):=\frac{1}{x^{2}+1}, \quad D_{2}(x):=\frac{x}{x^{2}+1} .
$$

The convergence strips of the Mellin transforms $\check{D}_{1}(z), \check{D}_{2}(z)$ are $-2<\Re z<0,-1<$ $\Re z<1$, respectively.

Choose $X>0$ so large that $f$ is finite and decreasing on $[X, \infty)$. We write $\tilde{f}(x):=I_{[X, \infty)} f(x)$. In the proof below we first prove

$$
\frac{\left(D_{2} * \tilde{f}\right)(x)}{\left(D_{1} * \tilde{f}\right)(x)} \rightarrow c \quad(x \rightarrow \infty)
$$

and then apply Theorems 1,2 and 3.

Step 1. By (2.8), both $F_{1}(x)$ and $F_{2}(x)$ are of constant sign for $x$ large enough. So by Theorem 36 of [26], $D_{2} * f=C * F_{2}$ :

$$
\int_{0}^{\infty} \frac{x}{x^{2}+t^{2}} f(t) d t=\int_{0}^{\infty} e^{-x / t} F_{2}(t) d t / t \quad(x>0)
$$

(the integrals are improper in the first instance but reduce to absolutely convergent ones since both $f(x)$ and $F_{2}(x)$ are of constant sign for $x$ large enough). In particular, together with (2.8), this gives

$$
\int_{a}^{\infty}\left|F_{i}(t)\right| d t / t<\infty \quad(i=1,2, \quad \forall a>0)
$$

Next we show $D_{1} * f=C * F_{1}$ :

$$
\int_{0}^{\infty} \frac{t}{x^{2}+t^{2}} f(t) d t=\int_{0}^{\infty} e^{-x / t} F_{1}(t) d t / t \quad(0<x<\infty),
$$

both integrals converging absolutely. We write $\tilde{F}_{\mathrm{s}}$ for the Fourier sine transform of $\tilde{f}$. Since (7.4) holds for the pair of $I_{(0, X)}(x) f(x)$ and its Fourier sine transform by Theorem 35 of [26], it is enough to prove

$$
\int_{0}^{\infty} \frac{t}{x^{2}+t^{2}} \tilde{f}(t) d t=\int_{0}^{\infty} e^{-x u} \tilde{F}_{\mathrm{B}}(u) d u \quad(0<x<\infty)
$$


in order to get (7.4).

By the second integral mean-value theorem,

$$
\left|\tilde{F}_{\mathrm{B}}(x)\right|=\left|\int_{X}^{\infty} f(t) \sin t x d t\right| \leq \frac{2 f(X+)}{x} \quad(0<x<\infty) .
$$

By residue calculus,

$$
\int_{0}^{M} \frac{t \sin t u}{x^{2}+t^{2}} d t=\frac{\pi}{2} e^{-x u}-R_{M}(u ; x) \quad(u, x, M>0)
$$

where

$$
R_{M}(u ; x):=\frac{1}{2} \Im\left(\int_{\gamma} \frac{z e^{i z u}}{z^{2}+x^{2}} d z\right)
$$

and $\gamma$ is the semicircle $z=M e^{i \theta}$ with $\theta$ increasing from 0 to $\pi$. For $M>\sqrt{2} x$,

$$
\left|R_{M}(u ; x)\right| \leq \int_{0}^{\pi} e^{-u M \sin \theta} d \theta \leq 2 \int_{0}^{\pi / 2} e^{-2 u M \theta / \pi} d \theta \leq \min \left(\pi, \frac{\pi}{M u}\right) .
$$

By Theorem 38 of [26], for $M$ large enough,

$$
\int_{0}^{M} \frac{t}{x^{2}+t^{2}} \tilde{f}(t) d t=\int_{0}^{\infty}\left\{e^{-x u}-\frac{2}{\pi} R_{M}(u ; x)\right\} \tilde{F}_{\mathbf{s}}(u) d u,
$$

where the integral on the right is improper both at 0 and $\infty$ in the first instance but reduces to an absolutely convergent one by (7.3), (7.6) and (7.7). These estimates also assure suitable dominated convergence in (7.8), as $M \rightarrow \infty$, to yield (7.5), whence (7.4). From (7.4), we have in particular

$$
\int_{X}^{\infty} \frac{f(t)}{t} d t<\infty
$$

Step 2. We prove (7.1). Choose $Y$ so large that $F_{1}(x)$ is of constant sign for $x>Y$. Then for $M \geq Y$ and $i=1,2$,

$$
\begin{gathered}
\frac{\int_{0}^{M} e^{-x / t}\left|F_{i}(t)\right| d t / t}{\int_{Y}^{\infty} e^{-x / t}\left|F_{1}(t)\right| d t / t} \leq \frac{\int_{0}^{M} e^{-x / t}\left|F_{i}(t)\right| d t / t}{\int_{M}^{\infty} e^{-x / t}\left|F_{1}(t)\right| d t / t} \\
\leq \frac{\int_{0}^{M} \exp \left\{-x\left(\frac{1}{t}-\frac{1}{M}\right)\right\}\left|F_{i}(t)\right| d t / t}{\int_{M}^{\infty}\left|F_{1}(t)\right| d t / t} \rightarrow 0 \quad(x \rightarrow \infty) .
\end{gathered}
$$

In particular,

$$
\frac{C * F_{1}(x)}{\int_{Y}^{\infty} e^{-x / t} F_{1}(t) d t / t}=1+\frac{\int_{0}^{Y} e^{-x / t} F_{1}(t) d t / t}{\int_{Y}^{\infty} e^{-x / t} F_{1}(t) d t / t} \rightarrow 1 \quad(x \rightarrow \infty) .
$$


For any $\epsilon>0$, we can find $M>Y$ so that

$$
=\left|\frac{F_{2}(x)}{F_{1}(x)}-c\right| \leq \epsilon \quad(x \geq M)
$$

Then

$$
\left|\frac{C * F_{2}(x)}{\int_{Y}^{\infty} e^{-x / t} F_{1}(t) d t / t}-c\right| \leq I(x)+I I(x)+I I I(x)
$$

where

$$
\begin{gathered}
I(x):=\frac{\int_{M}^{\infty} e^{-x / t}\left|F_{2}(t)-c F_{1}(t)\right| d t / t}{\int_{Y}^{\infty} e^{-x / t}\left|F_{1}(t)\right| d t / t} \\
I I(x):=\frac{\int_{0}^{M} e^{-x / t}\left|F_{2}(t)\right| d t / t}{\int_{Y}^{\infty} e^{-x / t}\left|F_{1}(t)\right| d t / t} \\
I I I(x):=\frac{c \int_{Y}^{M} e^{-x / t}\left|F_{1}(t)\right| d t / t}{\int_{Y}^{\infty} e^{-x / t}\left|F_{1}(t)\right| d t / t}
\end{gathered}
$$

Clearly, $I(x) \leq \epsilon$, while by (7.10) both $I I(x)$ and $I I I(x)$ tend to zero as $x \rightarrow \infty$. Combining,

$$
\frac{C * F_{2}(x)}{\int_{Y}^{\infty} e^{-x / t} F_{1}(t) d t / t} \rightarrow c \quad(x \rightarrow \infty)
$$

whence

$$
\frac{D_{2} * f(x)}{D_{1} * f(x)}=\frac{C * F_{2}(x)}{C * F_{1}(x)} \rightarrow c \quad(x \rightarrow \infty)
$$

Now we have

$$
\int_{0}^{\infty} f(t) d t=\infty
$$

For, if not, then $f \in L^{1}[0, \infty)$. However by (2.7) the dominated convergence theorem yields

$$
\frac{F_{\mathrm{c}}(x)}{F_{\mathrm{s}}(x)} \rightarrow \frac{0}{\int_{0}^{\infty} f(t) d t}=0 \quad(x \rightarrow 0+),
$$

contradicting (2.8). Thus (7.11) follows. Therefore

$$
\frac{D_{2} * f(x)}{D_{2} * \tilde{f}(x)}=1+\frac{\int_{0}^{X} f(t) d t /\left\{1+(t / x)^{2}\right\}}{\int_{X}^{\infty} f(t) d t /\left\{1+(t / x)^{2}\right\}} \rightarrow 1 \quad(x \rightarrow \infty) .
$$

Similarly,

$$
\frac{D_{1} * f(x)}{D_{1} * \tilde{f}(x)} \rightarrow 1 \quad(x \rightarrow \infty)
$$

Thus we obtain (7.1).

Step 3. Let $\rho$ be the upper order of $f$. In this step, we prove $-1<\rho<0$ and $c=$ 
$\tan (-\pi \rho / 2)$. Since $f$ is eventually decreasing, $\rho \leq 0$, while $\rho \geq-1$ by (7.11). Thus $-1 \leq \rho \leq 0$.

Now suppose $\rho=0$. For $\epsilon \in(0,1)$, let $E_{1}$ and $E_{2}$ be as in Step 3 of $\S 6$. Since

$$
D_{2} * \tilde{f}(x) \leq \int_{X}^{\infty} \frac{x}{2 x t} f(t) d t=\frac{1}{2} \int_{X}^{\infty} \frac{f(t)}{t} d t \quad(0<x<\infty),
$$

(7.9) shows that $D_{2} * \tilde{f}$ is bounded on $(0, \infty)$. Similarly, so is $D_{2} * \tilde{f}$. Therefore we may consider both $E_{2} * E_{1} * D_{1} * \tilde{f}$ and $E_{2} * E_{1} * D_{2} * \tilde{f}$. However, since

$$
\int_{0}^{1} \frac{D_{1}(t)}{t} d t=\infty, \quad \int_{0}^{\infty} \frac{D_{2}(t)}{t} d t<\infty
$$

arguing as in Step 3 of $\S 6$ we obtain a contradiction, whence $\rho \neq 0$.

Now since $-1 \leq \rho<0$ and

$$
\int_{0}^{\infty} D_{1}(t) d t<\infty, \quad \int_{0}^{\infty} D_{2}(t) d t=\infty
$$

we can apply Theorem 1 to obtain $\rho>-1$, whence $-1<\rho<0$.

Now we can apply Theorem 2 to obtain $c=\check{D}_{2}(\rho) / \check{D}_{1}(\rho)$. But

$$
\begin{aligned}
& \check{D}_{1}(z)=\int_{0}^{\infty} \frac{t^{-z-1}}{1+t^{2}} d t=\frac{(\pi / 2)}{\sin (-\pi z / 2)} \quad(-2<\Re z<0), \\
& \check{D}_{2}(z)=\int_{0}^{\infty} \frac{t^{-z}}{1+t^{2}} d t=\frac{(\pi / 2)}{\cos (\pi z / 2)} \quad(-1<\Re z<1),
\end{aligned}
$$

whence $c=\tan (-\pi \rho / 2)$.

Step 4. We write

$$
k_{0}(x):=\check{D}_{2}(\rho) D_{1}(x)-\check{D}_{1}(\rho) D_{2}(x) \quad(0<x<\infty) .
$$

Then

$$
\check{k}_{0}(z)=\frac{(\pi / 2)^{2}}{\sin (-\pi \rho / 2)} \frac{\{\tan (-\pi \rho / 2)-\tan (-\pi z / 2)\}}{\sin (-\pi z / 2)} \quad(-1<\Re z<0) .
$$

Now $\sin (-\pi z / 2)$ has no root in the strip $-1<\Re z<0$, while $\tan (-\pi z / 2)-\tan (-\pi \rho / 2)$ has a unique root $z=\rho$ in the strip and this root is simple. Therefore $\breve{k}_{0}(z)$ also has a unique root $z=\rho$ in this strip (whence on the line $\Re z=\rho$ ) and it is simple.

For any $r \in(-1,0)$ and any $\alpha>0$, we prove

$$
\frac{\log \left|\check{k}_{0}(r+i y)\right|}{\exp \left(\frac{\pi|y|}{2 \alpha}\right)} \rightarrow 0 \quad(y \rightarrow \pm \infty) .
$$


For $z=r+i y$,

$$
|\sin (-\pi z / 2)| \leq \frac{1}{2}\left(e^{\pi y / 2}+e^{-\pi y / 2}\right) \leq e^{\pi|y| / 2},
$$

while by the mean-value theorem

$$
|\sin (-\pi z / 2)| \geq \frac{1}{2}\left(e^{\pi|y| / 2}-e^{-\pi|y| / 2}\right) \geq \frac{\pi|y|}{2} e^{-\pi|y| / 2}
$$

whence

$$
|\log | \sin (-\pi z / 2)|| \leq \log (\pi / 2)+\pi|y|+|\log | y|| .
$$

On the other hand,

$$
\tan (-\pi z / 2) \rightarrow \mp i \quad(y \rightarrow \pm \infty)
$$

Thus (7.12) follows.

For small enough $\epsilon>0$, write $E_{2}(x):=I_{(0,1]}(x) x^{\rho+\epsilon}$ as above. Then we can apply Theorem 3 to obtain $E_{2} * D_{1} * \tilde{f} \in R_{\rho}$. As in Step 4 of $\S 6$, we can apply a monotone density argument to pass from $E_{2} * D_{1} * \tilde{f} \in R_{\rho}$ to $D_{1} * \tilde{f} \in R_{\rho}$. Now for $x>0$,

$$
\left(D_{1} * \tilde{f}\right)(\sqrt{x})=\int_{0}^{\infty} \frac{t \tilde{f}(t)}{x+t^{2}} d t=\frac{1}{2} \int_{0}^{\infty} \frac{\tilde{f}(\sqrt{s})}{x+s} d s=\frac{1}{2} \int_{(0, \infty)} \frac{d U(s)}{x+s}
$$

where

$$
U(x):=\int_{0}^{x} \tilde{f}(\sqrt{s}) d s=\int_{0}^{\sqrt{x}} t \tilde{f}(t) d t \quad(0<x<\infty) .
$$

Therefore by the Tauberian theorem for Stieltjes transforms (see, e.g., Theorem 1.7.4 of [2]), we obtain $U \in R_{(2+\rho) / 2}$. Again by a monotone density argument, $\tilde{f} \in R_{\rho}$, whence $f \in R_{\rho}$.

\section{$\S 8$. Counter-examples.}

We show, by counter-example, that Theorems 4 and 5 fail if the assumption $\int_{0}^{\infty} t^{\nu+\frac{1}{2}} f(t) d t \neq 0$ is not satisfied. Let $t^{\nu+\frac{1}{2}} f(t) \in L_{\text {loc }}^{1}[0, \infty), f$ be ultimately decreasing to zero at infinity with

$$
\int_{0}^{\infty} t^{\nu+\frac{1}{2}} f(t) d t=0
$$

We also assume

$$
f(t) \sim t^{\rho} l(t) \quad(t \rightarrow \infty)
$$

with $l \in R_{0}$ and

$$
-\nu-\frac{7}{2}<\rho<-\nu-\frac{3}{2}
$$


We have the following counter-example: for $M>0$ so large that $u^{\rho+\nu+\frac{1}{2}} l(u) \in L^{1}[M, \infty)$,

$$
f(t):=-\frac{\nu+\frac{3}{2}}{M^{\nu+\frac{3}{2}}} \int_{M}^{\infty} u^{\rho+\nu+\frac{1}{2}} l(u) d u \quad(0 \leq t \leq M), \quad:=t^{\rho} l(t) \quad(M<t<\infty) .
$$

Write

$$
g_{1}(x):=x^{-\nu-\frac{1}{2}} \int_{x}^{\infty} u^{\nu+\frac{1}{2}} f(u) d u \quad(x>0)
$$

Then $t^{(\nu+1)+\frac{1}{2}} g_{1}(x) \in L_{\text {loc }}^{1}[0, \infty), g_{1}$ is eventually decreasing to zero at infinity, and

$$
g_{1}(t) \sim t^{\rho+1} l(t) \cdot \frac{1}{-\left(\rho+\nu+\frac{3}{2}\right)} \quad(t \rightarrow \infty) .
$$

Let $F_{\nu}$ be the Hankel transform of $f$ (of Mellin convolution form). Since

$$
\frac{d}{d t}\left\{t^{-\nu} J_{\nu}(t)\right\}=-t^{-\nu} J_{\nu+1}(t)
$$

integration by parts yields

$$
\begin{gathered}
F_{\nu}(x)=x^{-\nu-\frac{3}{2}} \int_{0}^{\infty-} t^{\nu+\frac{1}{2}} f(t)(t / x)^{-\nu} J_{\nu}(t / x) d t \\
=-\frac{1}{x} \cdot \frac{1}{x} \int_{0}^{\infty} g_{1}(t)(t / x)^{1 / 2} J_{\nu+1}(t / x) d t \\
\sim x^{\rho} l(x) a_{\nu}(\rho) \quad(x \rightarrow \infty),
\end{gathered}
$$

where in the last step we used the Abelian theorem for Hankel transforms of order $\nu+1$ and the equality

$$
\frac{a_{\nu+1}(\rho+1)}{\left(\rho+\nu+\frac{3}{2}\right)}=a_{\nu}(\rho)
$$

Therefore

$$
\frac{F_{\nu}(x)}{f(x)} \rightarrow a_{\nu}(\rho)<0 \quad(x \rightarrow \infty)
$$

whence we have a counter-example.

One may not be satisfied with the example above because the limit $a_{\nu}(\rho)$ above is negative. So we consider another example with positive limit. Let $f$ be as above but with

$$
-\nu-\frac{11}{2}<\rho<-\nu-\frac{7}{2}
$$

instead of (8.3). We assume also

$$
\int_{0}^{\infty} t^{\nu+\frac{5}{2}} f(t) d t=0
$$


in addition to (8.1). For example, we obtain such $f$ if we choose $M$ large enough and $a_{0}$, $a_{1}$ suitably in

$$
f(t):=a_{0}+a_{1} t \quad(0 \leq t \leq M), \quad:=t^{\rho} l(t) \quad(M<t<\infty) .
$$

Let $g_{1}$ be as above. We write

$$
g_{2}(x):=x^{-\nu-\frac{3}{2}} \int_{x}^{\infty} t^{\nu+\frac{3}{2}} g_{1}(t) d t \quad(x>0) .
$$

Then $t^{(\nu+2)+\frac{1}{2}} g_{2}(x) \in L_{\text {loc }}^{1}[0, \infty), g_{2}$ is eventually decreasing to zero at infinity, and

$$
g_{2}(t) \sim t^{\rho+2} l(t) \cdot \frac{1}{\left(\rho+\nu+\frac{3}{2}\right)\left(\rho+\nu+\frac{7}{2}\right)} \quad(t \rightarrow \infty)
$$

Since

integration by parts yields

$$
\int_{0}^{\infty} t^{\nu+\frac{3}{2}} g_{1}(t) d t=\frac{1}{2} \int_{0}^{\infty} u^{\nu+\frac{5}{2}} f(u) d u=0
$$

$$
\begin{gathered}
F_{\nu}(x)=-x^{-\nu-\frac{7}{2}} \int_{0}^{\infty-} t^{\nu+\frac{3}{2}} g_{1}(t)(t / x)^{-\nu-1} J_{\nu+1}(t / x) d t \\
=\frac{1}{x^{2}} \\
\cdot \frac{1}{x} \int_{0}^{\infty} g_{2}(t)(t / x)^{1 / 2} J_{\nu+2}(t / x) d t \\
\sim x^{\rho} l(x) a_{\nu}(\rho) \quad(x \rightarrow \infty),
\end{gathered}
$$

where in the last step we used the Abelian theorem for Hankel transforms of order $\nu+2$ and the equality

$$
\frac{a_{\nu+2}(\rho+2)}{\left(\rho+\nu+\frac{3}{2}\right)\left(\rho+\nu+\frac{7}{2}\right)}=a_{\nu}(\rho)
$$

Therefore

$$
\frac{F_{\nu}(x)}{f(x)} \rightarrow a_{\nu}(\rho)>0 \quad(x \rightarrow \infty)
$$

whence we have a counter-example with positive limit.

By a similar method, one may construct a counter-example to show that Theorem 6 fails if the condition $\int_{0}^{\infty} f(t) d t \neq 0$ is not satisfied.

\section{References}

[1] N. H. BINGHAM, 'Probability theory on groups', Probability Measures on Groups IX, Lecture Notes in Math. 1379 (1989), 6-20, Springer. 
[2] N. H. BINGHAM, C. M. GOLDIE and J. L. TEUGELS, Regular variation (Encycl. Math. Appl. 27, 2nd ed., Cambridge Univ. Press, 1989; 1st ed. 1987).

[3] N. H. BINGHAM and A. INOUE, 'The Drasin-Shea-Jordan theorem for Fourier and Hankel transforms', Quart. J. Math. 48 no. 4 (1997), to appear (Statistics Report 95/1, Birkbeck College, University of London).

[4] A. A. BORICHEV, 'Beurling algebras and the generalized Fourier transform', Proc. London Math. Soc. (3) 73 (1996), 431-480.

[5] D. DRASIN, 'Tauberian theorems for slowly varying functions', Trans. American Math. Soc. 133 (1968), 333-356.

[6] D. DRASIN and D. F. SHEA, 'Complements to some theorems of Bowen and Macintyre on the radial growth of entire functions with negative zeros', Mathematical essays dedicated to A. J. Macintyre 101-121, Ohio Univ. Press, Athens, Ohio, 1970.

[7] D. DRASIN and D. F. SHEA, 'Convolution inequalities, regular variation and exceptional sets', J. Analyse Math. 29 (1976), 232-293.

[8] A. EDREI and W. H. J. FUCHS, 'Tauberian theorems for a class of meromorphic functions with negative zeros and positive poles', Contemporary problems in the theory of analytic functions 339-358, Nauka, Moscow, 1966.

[9] W. FELLER, An introduction to probability theory and its applications, Volume 2, 2nd edition, Wiley, 1971.

[10] T. GANELIUS, 'Regularly varying functions and Poincaré's theorem on difference equations', Symposia on Theor. Physics and Math. vol. 10 (Inst. Math. Sci., Madras, 1969), 7-17, Plenum. Press, New York, 1970 (Mathematical Reviews 41, $\sharp 8923$ ). 
[11] V. P. GURARII, 'Harmonic analysis in spaces with a weight', Trans. Moscow Math. Soc. (1979), 21-75.

[12] G. S. JORDAN, 'Regularly varying functions and convolutions of functions with real kernels', Trans. American Math. Soc. 194 (1974), 177-194.

[13] B. I. KORENBLUM, 'On a normed ring of functions with convolution' (in Russian), Dokl. Akad. Nauk SSSR (N.S.) 115 (1957), 226-229 (Mathematical Reviews 19 (1958), 968-9, E. Hewitt).

[14] B. I. KORENBLUM, 'A generalization of Wiener's Tauberian theorem and harmonic analysis of functions with rapid growth' (in Russian), Trud. Moscov. Mat. Obšč 7 (1958), 121-148.

[15] B. Ya. LEVIN, Distribution of zeros of entire functions, Translations of Math. Monographs 5, American Math. Soc., Providence, RI, 1964.

[16] W. MAGNUS, F. OBERHETTINGER and R. P. SONI, Formulas and theorems for the special functions of mathematical physics, 3rd ed. (Springer, Berlin, 1966).

[17] B. NYMAN, On the one-dimensional translation group and semigroup in certain function spaces (Appelbergs Boktryckeri, Uppsala, 1950) (Mathematical Reviews 12 (1951), 108-109, J. Korevaar).

[18] R. E. A. C. PALEY and N. WIENER, 'Notes on the theory and applications of Fourier transforms VII: On the Volterra equation', Trans. American Math. Soc. 35 (1933), 785-791.

[19] R. E. A. C. PALEY and N. WIENER, Fourier transforms in the complex domain, Colloquium Publications 19, American Math. Soc., Providence, RI, 1934. 
[20] E. J. G. PITMAN, 'On the behaviour of the characteristic function of a probability distribution in the neighbourhood of the origin', J. Australian Math. Soc. Series A 8 (1968), 422-443.

[21] H. R. PITT, Tauberian theorems, Oxford University Press, 1958.

[22] H. RADEMACHER, Topics in analytic number theory (Springer, Berlin, 1973).

[23] H. REITER, Classical harmonic analysis and locally compact groups (Oxford University Press, 1968).

[24] D. F. SHEA, 'A complement to Valiron's Tauberian theorem for the Stieltjes transform', Proc. American Math. Soc. 21 (1969), 1-9.

[25] E. C. TITCHMARSH, The theory of functions, 2nd ed. (Oxford University Press, 1939).

[26] E. C. TITCHMARSH, Theory of Fourier integrals, 2nd ed. (Oxford University Press, 1948).

[27] G. N. WATSON, Theory of Bessel functions, 2nd ed. (Cambridge University Press, 1944).

[28] E. C. WHITTAKER and G. N. WATSON, Modern analysis, 4th ed. (Cambridge University Press, 1948).

Department of Statistics, Birkbeck College (University of London), Malet Street, London WC1E 7HX

Department of Mathematics, Faculty of Science, Hokkaido University, Sapporo 060, Japan 